\title{
Kommunale Online-Partizipation - Wer ist gefragt?
}

\section{Communal online participation - who may take part?}

\section{Frank Dietrich, Düsseldorf \& Jonathan Seim, Düsseldorf}

Zusammenfassung: Das Internet bietet die Möglichkeit, eine beliebig große Anzahl von Personen durch unterschiedliche Formen der Deliberation und Beschlussfassung politisch einzubinden. Insbesondere im kommunalen Kontext wird die Online-Partizipation - etwa im Rahmen städtischer Bürgerhaushalte - bereits vielfach als Mittel erprobt, um die soziale Akzeptanz und Legitimität politischer Entscheidungen zu erhöhen. Die Legitimität demokratischer Verfahren hängt neben anderen Faktoren maßgeblich von der Konstitution des Demos und der damit festgelegten Allokation der Teilnahmerechte ab. In historischer Perspektive hat vor allem die Exklusion bestimmter Gruppen, insbesondere von Frauen, Besitzlosen oder nichtweißen Personen, die Legitimität klassischer Offline-Verfahren, wie z.B. Parlamentswahlen, in Frage gestellt. Die neuen Formen der Online-Partizipation bergen hingegen - so die zentrale These des Aufsatzes - die Gefahr der Über-Inklusivität, weil sie häufig auf jegliche Zugangsbeschränkung verzichten. Selbst wenn die Verfahren, wie in der Praxis üblich, einen konsultativen Charakter haben und die politischen Entscheidungsträger formal nicht binden, führt ihre Offenheit zu Legitimationsproblemen. Daraus entsteht die Notwendigkeit, normative Kriterien für die Vergabe von Partizipationsrechten zu entwickeln, die den Besonderheiten der Online-Deliberation angemessen Rechnung tragen.

Schlagwörter: Bürgerbeteiligung, Demokratie, Demos, Internet, Legitimität

Abstract: The Internet allows for the involvement of any number of people in various forms of political deliberation and decision-making. Especially in the municipal context, online participation, for example participatory budgeting, is often implemented as a means of increasing the social acceptance of political decisions. The legitimacy 
of democratic procedures importantly depends, among other factors, on the constitution of the demos and the concomitant allocation of participation rights. Historically, the exclusion of certain groups, in particular women, the dispossessed or people of color, has called into question the legitimacy of classic offline procedures, such as parliamentary elections. However, new forms of online participation introduce - according to the central thesis of this essay - the risk of over-inclusiveness, because they often dispense with any kind of access restriction. Although, the procedures typically are consultative in nature and do not formally bind the political decision-makers, their unrestricted accessibility poses problems of legitimacy. Consequently, there is a need to develop normative criteria for the allocation of participation rights, which take the specifics of municipal online participation into account.

Keywords: Citizen Participation, Democracy, Demos, Internet, Legitimacy

\section{Einleitung}

Für die Funktionsfähigkeit moderner Demokratien stellen politisches Desinteresse und mangelndes Engagement der Bürgerinnen und Bürger eine ernst zu nehmende Gefahr dar. Fehlende Möglichkeiten der Partizipation und die Intransparenz politischer Entscheidungsprozesse werden häufig als Ursachen für „Politikverdrossenheit“ genannt. Das Internet bietet die Chance, neue Formen der Beteiligung zu etablieren, die die Bürgerinnen und Bürger stärker in die demokratische Deliberation und Beschlussfassung einbinden. Mit der Ergänzung herkömmlicher Verfahren durch Elemente einer „virtuellen Demokratie“ verbindet sich die Hoffnung, die Identifikation mit dem politischen System zu fördern und die Akzeptanz politischer Entscheidungen zu erhöhen. Zudem verspricht die Online-Partizipation, demokratischen Verfahren mehr Legitimität zu verleihen und sie dem Ideal der Selbstregierung näher zu bringen. In jüngster Zeit haben vor allem die Städte eine Vorreiterrolle bei der Erprobung unterschiedlicher internetbasierter Teilhabeinstrumente eingenommen. Der kommunale Bereich hat sich zu einem wichtigen Experimentierfeld entwickelt, in dem die Bürger über vielfältige Optionen der demokratischen Mitwirkung verfügen.

Die kommunale Online-Partizipation wirft jedoch grundlegende demokratietheoretische Fragen auf, die einer eingehenden Erörterung bedürfen. Die erwarteten Vorteile der virtuellen Teilhabe werden sich nur dann einstellen, wenn die eingesetzten Verfahren wichtigen Legitimitätsstandards genügen. Die Legitimität partizipativer Online-Verfahren hängt - neben anderen Faktoren - ganz wesentlich von der Regelung des Zugangs ab. Der 
vorliegende Beitrag befasst sich daher mit der Vergabe politischer Partizipationsrechte, von der die Befugnis zur Mitwirkung an Beteiligungsverfahren abhängt. Die kommunale Online-Partizipation sieht sich bei der Bestimmung des zur Teilnahme befugten Personenkreises mit anderen Problemen konfrontiert als herkömmliche Offline-Verfahren. Während traditionell der Ausschluss bestimmter Bevölkerungsgruppen Anlass zu Kritik gegeben hat, ruft heute - so die zentrale These - die große Offenheit virtueller Teilhabeinstrumente Zweifel an ihrer Legitimität hervor.

Die Untersuchung untergliedert sich in vier Teile: Im folgenden Abschnitt gilt es zunächst, die Bedeutung der städtischen Demokratie zu erläutern und die spezifische Erscheinungsform der kommunalen Online-Partizipation darzulegen (2). Anschließend werden - auf einer grundsätzlichen Ebene - die demokratietheoretischen Fragen erörtert, die die Vergabe von Partizipationsrechten und somit die Konstitution der politischen Gemeinschaft aufwirft (3). Darauf aufbauend werden die Besonderheiten der kommunalen Online-Partizipation in den Blick genommen und die Über-Inklusivität, d.h. die potenzielle Teilnahme von Personen, die dem Demos nicht sinnvoll zugerechnet werden können, als zentrales Legitimationsproblem ausgewiesen (4). Sodann werden mögliche Einwände gegen die hier vertretene Position diskutiert, die sich aus dem konsultativen Charakter kommunaler Online-Instrumente ergeben (5). Die wichtigsten Ergebnisse der Argumentation werden abschließend kurz resümiert (6).

\section{Kommunale Online-Partizipation}

Der Zusammenhang von Stadt und Demokratie hat sich über die letzten Jahrtausende sowohl ideengeschichtlich als auch politisch stark verändert. Das Ideal einer vom Volk selbst ausgehenden politischen Herrschaft wurde erstmals in den griechischen Stadtstaaten der Antike in die Praxis umgesetzt. Zumindest für Aristoteles (2012, 1326b) stand dabei fest, dass sich Staatlichkeit und erst recht Demokratie nur in einem überschaubaren Stadtstaat realisieren lassen. ${ }^{1}$ Denn unter Demokratie verstand er die direkte Beteiligung an politischen Entscheidungen im Rahmen öffentlicher Versammlungen, was der Größe des Stadtgebietes und der Anzahl der Bürger natürliche

$1 \quad$ Entgegen dem modernen Sprachgebrauch hat Aristoteles (2012, 1279b) eine „Herrschaft der Vielen“, die dem Gemeinwohl verpflichtet ist, als Politie bezeichnet und den Begriff der Demokratie für die entartete Form, die nur den Interessen der Herrschenden dient, verwendet. 
Grenzen auferlegte. Ähnliche Vorstellungen finden sich noch Ende des 18. Jahrhunderts prominent bei Rousseau $(2011,57)$, der die Auffassung vertrat, dass nur diejenige politische Gemeinschaft zur gemeinsamen Gesetzgebung geeignet sei, in der jedes Mitglied allen anderen bekannt sein könne. Vor diesem Hintergrund standen während der Verfassungsdebatte in Nordamerika 1787/88 die Befürworter eines einheitlichen Bundesstaates vor der theoretischen Herausforderung, erklären zu müssen, wie eine Selbstregierung des Volkes und der Schutz der Freiheit in einem großen und bevölkerungsreichen Flächenstaat denkbar seien. In den sogenannten Federalist Papers argumentierten sie, dass Ersteres in ausreichendem Maße durch das Instrument der politischen Repräsentation möglich und Zweiteres gerade in einem großen, pluralen Staat, in welchem keine Parteiung mit einer dauerhaften Mehrheit rechnen könne, gewährleistet sei (Hamilton et al. 2007, Nr. 10, 63). Die Befürworter des Bundesstaates setzten sich damals durch - der Nationalstaat erscheint heute als natürlicher Ort und das repräsentative System als adäquate Institutionalisierung der Demokratie.

Nun muss man weder antiken Idealen anhängen, welche schon zu Rousseaus Zeiten etwas anachronistisch anmuteten, noch repräsentative Systeme strikt ablehnen, um den Bedeutungsverlust unmittelbarer politischer Partizipation zu bedauern. Die vorgebrachten Gründe für den Wert politischer Partizipation sind zahlreich und können hier nur angedeutet werden. Nach Auffassung einiger Theoretiker besteht eine enge kausale Verknüpfung zwischen dem politischen Engagement der Bürgerinnen und Bürger und bürgerschaftlichen Tugenden, die für eine gelingende Demokratie unerlässlich sind (Pateman 1970). So liege in dem Mangel effektiver Beteiligungsmöglichkeiten eine wichtige Ursache für zentrale Probleme moderner Demokratien wie politische Apathie und Politikverdrossenheit der Bürgerinnen und Bürger (Barber 2009). Die Lösung wird oftmals in der teilweisen Ersetzung oder zumindest Ergänzung repräsentativer Institutionen durch Formen unmittelbarer Partizipation gesehen. In Anlehnung an Tocqueville (2014) wird davon ausgegangen, dass sich die Menschen durch ihre Teilhabe an der Regelung der öffentlichen Angelegenheiten zu interessierten und engagierten Mitgliedern des jeweiligen demokratischen Gemeinwesens entwickeln (Barber 2009, 153). Auf ähnliche Weise wird die Bedeutung der durch Partizipation angeeigneten Tugenden für den demokratischen Diskurs (Cooke 2000) und die Funktionsfähigkeit einer kritischen und die Regierung kontrollierenden Öffentlichkeit (Crouch 2004) betont. Anderen zufolge führt der kommunikative Austausch zwischen Gesellschaft und Staat, 
der auch durch Bürgerbeteiligung institutionalisiert wird, zu in verschiedener Hinsicht besseren Ergebnissen politischer Entscheidungsfindung (Habermas 1992). Spätestens seit den Protestbewegungen der 1960er und 7oer Jahre wird die Ergänzung des repräsentativen Systems um unmittelbare Einflussmöglichkeiten auch von den Bürgerinnen und Bürgern immer mehr eingefordert. Zugleich ist auf politischer Ebene spätestens seit den 1990er Jahren die Bereitschaft gewachsen, dem „Wahlvolk“ mehr Gelegenheit zu Mitwirkung in demokratischen Entscheidungsprozessen zu geben.

Im Kontext der partizipativen Bestrebungen, die in vielen modernen Demokratien zu beobachten sind, fungiert die Stadt als bevorzugte Arena demokratischer Innovationen. Der kommunale Kontext bietet verschiedene Vorteile für die Etablierung demokratischer Mitwirkungsinstrumente, die z.T. auf eine lange theoriegeschichtliche Tradition zurückblicken. Wie schon Tocqueville $(2014,71,245)$ bemerkte, ist auf lokaler Ebene die Verbindung zwischen den Interessen der Individuen und dem politischen Handeln der Gemeinschaft besonders eng und die Bedeutung politischen Engagements für die Gestaltung des eigenen unmittelbaren Lebensumfeldes besonders deutlich. Zudem lässt sich die - zu weiteren Aktivitäten motivierende - Erfahrung, durch eigenes Engagement politisch etwas bewirken zu können, tendenziell eher in überschaubaren Gruppen machen. Ferner empfiehlt sich die Stadt, wie bereits Aristoteles hervorgehoben hat, auch deshalb als Ort demokratischer Selbstbestimmung, weil die diskursive Funktionsfähigkeit öffentlicher Versammlungen stark von der Größe der politischen Gemeinschaft abhängt (Kreager 2008, 618). Insgesamt scheint die Stadt, auch wenn sie mit dem antiken Stadtstaat kaum zu vergleichen ist, im modernen Nationalstaat nicht von der politischen Landkarte zu verschwinden, sondern ganz im Gegenteil als Ort demokratischer Partizipation wiederentdeckt zu werden.

An der demokratietheoretischen Bedeutung des kommunalen Bereichs hat die Erfindung und Verbreitung des Internets nicht prinzipiell etwas geändert. Auch wenn das Internet die Zusammenkunft vieler Menschen aus aller Welt ermöglicht und diese immer mehr wie ein „globales Dorf“ (McLuhan und Powers 1992) erscheinen lässt, hebelt es die vorstehend skizzierten Vorteile kleiner Gruppen nicht vollständig aus. Denn selbst im digitalen Raum müssen diskursive Verfahren, welche minimalen Qualitätsstandards genügen und z.B. der Stimme jedes Diskursteilnehmers Gehör verschaffen wollen, die Anzahl der mitwirkenden Personen limitieren. Das Internet bietet aber, auch wenn man die allzu euphorischen Erwartungen der Anfangszeit 
hinter sich lässt, ein enormes demokratisches Potenzial. Ein wesentlicher Vorteil der Online-Partizipation im Vergleich zu klassischen Offline-Verfahren liegt in dem weitaus geringeren Aufwand, der mit der Teilnahme verbunden ist. Zudem ermöglicht der digitale Raum die asynchrone und flexible Beteiligung einer Vielzahl von Personen über einen längeren Zeitraum hinweg. Da die Bereitschaft der Individuen zur politischen Mitwirkung maßgeblich von den zur Verfügung stehenden Zeitressourcen abhängt (Brady, Verba und Schlozman 1995), können internetbasierte Partizipationsinstrumente einen wichtigen Beitrag zu demokratischer Aktivierung leisten.

Wirft man nun einen Blick auf die politische Wirklichkeit der Demokratie in Deutschland, dann fällt auf, dass die Städte auch de facto eine besondere Rolle spielen. Während bis heute auf Bundesebene keine direktdemokratischen Instrumente vorgesehen sind, sind solche seit den späten 9oer Jahren in den Landesverfassungen aller deutschen Länder verankert und werden vor allem in den Kommunen genutzt. Neben den gesetzlich geregelten Verfahren werden insbesondere von Städten zunehmend informelle Beteiligungsverfahren praktiziert, die in den Gemeindeordnungen der Länder nicht näher bestimmt sind. Über ihre Initiierung und Ausgestaltung entscheiden die Städte selbst auf freiwilliger Basis. Auch wenn die Kommunen zur Durchführung solcher Verfahren rechtlich nicht verpflichtet sind, scheint die wachsende Einbindung der Bürger in staatliche Entscheidungsprozesse mittlerweile im Selbstverständnis der Städte und Kommunen tief verankert zu sein:

Die Beteiligung von Bürgerinnen und Bürgern an der Stadtentwicklung in unterschiedlichen Formen gehört längst zur selbstverständlichen kommunalen Praxis. (Deutscher Städtetag 2013a, 5)

Die Kommunen haben sich in den letzten Jahrzehnten zu Laboratorien der Bürgerbeteiligung entwickelt, insbesondere was Eigeninitiative in Form nicht gesetzlich geregelter, informeller Beteiligungsverfahren anlangt. (Deutscher Städtetag 2013b, 3)

Als besonderer Ausdruck solcher Bekenntnisse zu mehr demokratischer Teilhabe lassen sich die sogenannten Leitlinien zur Bürgerbeteiligung lesen, die in den letzten Jahren in vielen Städten verabschiedet wurden. Die Leitlinien geben Standards für die Durchführung von Partizipationsverfahren vor und stellen ihren vermehrten Einsatz in Aussicht. Im Zuge der Bürgerbeteiligungsverfahren erfreut sich das Internet zunehmender Beliebtheit - so 
haben etwa in NRW bereits bis 2016 ein Drittel der Kommunen und drei Viertel der Großstädte Erfahrungen mit Online-Bürgerbeteiligung gemacht (Wilker et al. 2016). Beispiele hierfür sind die sogenannten Bürgerbudgets, bei denen es um die Nutzung eines von der Stadt vorgegebenen Geldbetrags geht, und Raddialoge, die sich der Verbesserung der städtischen Radinfrastruktur widmen. In beiden Fällen können über Internetseiten Vorschläge eingereicht, kommentiert und bewertet bzw. unterstützt werden (Ruesch 2018; Escher 2018).

Im Bereich der informellen Partizipation verfügen die Kommunen über einen weiten Gestaltungsspielraum, den sie zur Initiierung verschiedenartiger Beteiligungsverfahren nutzen. Erstens werden aggregative und deliberative Elemente (,voice“ und „vote“) in den praktizierten Verfahren der Bürgerbeteiligung sehr unterschiedlich eingesetzt. Während z.B. bei Bürgerbudgets in der Regel die Abstimmung über spezifische Projektideen im Zentrum steht, geht es bei anderen Verfahren primär um die gemeinsame Diskussion und Erarbeitung von Vorschlägen. Zweitens werden die Teilnehmenden in unterschiedlicher Form und mit unterschiedlicher Intensität in die politischen Entscheidungsprozesse einbezogen. Dies wird in der Fachliteratur mit einem Kontinuum beschrieben, das von der reinen Information der Bevölkerung an dem einen Ende über die Konsultierung bis hin zur vollkommenen Übernahme der von den Bürgerinnen und Bürgern getroffenen Entscheidung am anderen Ende reicht (Arnstein 1969; IAP2 2018). Drittens variieren die Gegenstände der Verfahren bezüglich ihrer Nähe zu konkreten Entscheidungen. Während es bei einigen Verfahren um sehr konkrete Sachfragen z. B. bezüglich der Gestaltung des öffentlichen Raumes geht, werden im Rahmen sogenannter Zukunftswerkstätten sehr allgemeine Leitbilder entwickelt, deren Handlungsempfehlungen oftmals recht abstrakt bleiben. Schließlich werden die digitalen Partizipationsverfahren, die in den Städten zur Anwendung gelangen, z.T. mit verschiedenartigen Offline-Elementen kombiniert. So gibt es mitunter die Möglichkeit, zusätzlich zu Online-Foren auch an Bürgerversammlungen in Präsenz teilzunehmen oder den eigenen Beitrag auf postalischem Wege einzubringen.

Der vorliegende Aufsatz nimmt grundsätzlich alle kommunalen Partizipationsverfahren in den Blick, die ein starkes Online-Element beinhalten und sich insofern gleichermaßen mit den nachstehend zu erörternden Legitimationsfragen konfrontiert sehen. Vorausgesetzt wird allerdings, dass die Beteiligungsinstrumente von den Kommunen selbst und nicht etwa von zivilgesellschaftlichen Akteuren initiiert werden. Für zivilgesellschaftliche 
Formen der Partizipation, die im städtischen Raum praktiziert werden, gelten andere und mutmaßlich schwächere Legitimitätsanforderungen. So scheint es z. B. unproblematisch zu sein, wenn der Ortsverband einer Partei oder ein Verein die Teilnahme von vornherein auf die eigenen Mitglieder beschränkt. Die von den Kommunen selbst initiierten Formen der Online-Partizipation unterliegen zwei Beschränkungen. Zum einen bleiben Verfahren unberücksichtigt, die sich nicht primär an die Bürgerschaft, sondern an Interessenvertreter oder Fachleute, z. B. in Form von Experten-Hearings, richten. Zum anderen werden Verfahren nicht betrachtet, die einseitig der Information dienen und den Bürgerinnen und Bürgern keine Möglichkeit einräumen, auf die politischen Entscheidungsträger einzuwirken. Da es in beiden Fällen offenbar nicht darum geht, die (gesamte) Bürgerschaft an dem Zustandekommen politischer Entscheidungen teilhaben zu lassen, sind sie nicht Gegenstand der weiteren Überlegungen.

Die kommunalen Beteiligungsverfahren, die im Fokus der vorliegenden Untersuchung stehen, weisen zwei Charakteristika auf, die für die weitere legitimationstheoretische Diskussion besondere Relevanz haben. Erstens sind die Resultate der Online-Partizipation rechtlich unverbindlich, d.h., die formale Entscheidungskompetenz verbleibt bei staatlicher Stelle. Die oftmals geäußerte Zusicherung einer besonderen Berücksichtigung der Ergebnisse lässt einen großen Spielraum in der praktischen Umsetzung und stellt eine letztlich nicht einklagbare Selbstverpflichtung dar. Zweitens ist der Zugang zu virtuellen Teilhabeinstrumenten in der Regel nicht auf die Einwohner der jeweiligen Kommune beschränkt. ${ }^{2}$ Auch bei Verfahren, die primär die städtische Bevölkerung adressieren, wird der Wohnsitz bei der Registrierung für die jeweilige Online-Plattform - sofern überhaupt notwendig - oftmals nicht abgefragt. Selbst in den wenigen Fällen, wo die Angabe des Wohnortes erforderlich ist, werden die Daten nicht überprüft, weshalb sich faktisch jeder registrieren und beteiligen kann (Masser, Pistoia und Nitzsche 2013, 103).

2 In den Leitlinien für kommunale Bürgerbeteiligung wird die Frage des Adressatenkreises oftmals gar nicht angesprochen oder sogar explizit auf eine Begrenzung der Teilnehmer verzichtet. So ist Bürgerbeteiligung in Berlin für alle Interessierten und in Jena für alle, die sich beteiligen wollen, geöffnet (Senatsverwaltung für Stadtentwicklung und Wohnen 2019, 17; Stadt Jena 2016, 8). In den Leitlinien anderer Städte werden zwar bestimmte Gruppen, wie Bürger oder Einwohner, besonders adressiert, ohne jedoch die Partizipation anderer Personen ausdrücklich auszuschließen. 


\section{Virtuelle Teilhaberechte}

Die Hoffnung, die Demokratie durch kommunale Online-Partizipation zu stärken, kann sich nur erfüllen, wenn die praktizierten Verfahren basalen Legitimitätsbedingungen genügen. Die Konzeptionierung wie auch die Implementierung partizipativer Online-Instrumente sieht sich jedoch vor erhebliche Herausforderungen gestellt. Bei der Implementierung hat sich in den letzten Jahren zum einen gezeigt, wie schwierig es ist, die Bürger und Bürgerinnen zur Teilnahme an kommunalen Online-Verfahren zu bewegen. So war z. B. das Interesse an den Bürgerhaushalten, die eine beachtliche Zahl von Gemeinden erprobt haben, in den meisten Fällen gering (Zepic, Dapp und Krcmar 2017). Zum anderen sind die Fähigkeit und die Bereitschaft, das Internet zur politischen Mitwirkung zu nutzen, in der Bevölkerung sehr unterschiedlich ausgeprägt. Ältere Menschen nehmen z. B. weit weniger an kommunalen Online-Verfahren teil als Angehörige der jüngeren Generation, für die virtuelle Formen der Kommunikation zum Alttag gehören (Masser, Pistoia und Nitzsche 2013, 85-92). Beide Phänomene - die geringe Teilnahme und die ungleiche Repräsentation der Bevölkerung - wecken Zweifel, ob in partizipativen Online-Verfahren tatsächlich der „Volkswille“ zum Ausdruck kommt.

Das demokratische Potenzial kommunaler Online-Partizipation kann sich nur dann entfalten, wenn es gelingt, solche und weitere Schwierigkeiten, die sich bei der Durchführung der Verfahren in der Praxis zeigen, zu überwinden. Kommunale Online-Verfahren begegnen auf der konzeptionellen Ebene allerdings noch grundlegenderen Herausforderungen, an denen ihre Legitimität scheitern kann. Von besonderer Bedeutung und wenig beachtet ist hierbei die Regelung der Zugangsbedingungen, die darüber entscheidet, wer die Möglichkeit zur Mitsprache erhält bzw. auf sie verzichten muss. Verfahren der kommunalen Online-Partizipation können nur dann als legitim gelten, wenn sie den Kreis der zur Teilnahme befugten Personen in angemessener Weise bestimmen.

Die Frage nach der Zusammensetzung der maßgeblichen Abstimmungs- oder Beratungsgemeinschaft wird in der Demokratietheorie als „Demos-Problem" bezeichnet. Sie ist erst relativ spät - ab den 1970er Jahren - durch die Arbeiten von Robert Dahl (1989; 1990) in den Fokus gerückt. Laut Dahl (1989, 119-122) reichen prozedurale Kriterien nicht aus, um zu entscheiden, ob ein politisches Gemeinwesen als Demokratie klassifiziert werden kann. Seiner Auffassung nach kann ein Staat ,undemokratisch“ sein, obwohl er die Stimmen der Wahlberechtigten gleich gewichtet und Beschlüs- 
se durch Mehrheitsverfahren herbeiführt. Das südafrikanische Apartheidsregime habe z.B. den prozeduralen Bedingungen einer Demokratie genügt, aber einem Großteil der Bevölkerung das Wahlrecht vorenthalten. Es könne nicht im eigentlichen Sinne als Demokratie gelten, weil es die nichtweiße Bevölkerung in unzulässiger Weise aus der politischen Gemeinschaft ausgegrenzt habe. Dahl zufolge verdient ein politisches Gemeinwesen nur dann als Demokratie bezeichnet zu werden, wenn es die Zugehörigkeit zum Demos korrekt bestimmt.

Die Festlegung der Personengruppe, die zur Teilnahme an demokratischen Entscheidungen berechtigt ist, stellt die Demokratietheorie jedoch vor erhebliche Probleme. Offenkundig kann mit Hilfe von Verfahren, die den vorstehend angesprochenen prozeduralen Standards genügen, kein Aufschluss über die Zusammensetzung des Demos gewonnen werden. Ein demokratischer Mehrheitsbeschluss über die Vergabe von Teilhaberechten würde - auf höherer Ebene - erneut die Frage aufwerfen, wer stimmberechtigt ist. Wenn in einer Gesellschaft z. B. die Ausgrenzung von nichtweißen Personen aus dem politischen Prozess angefochten wird, dürfte auch die Befugnis des weißen Bevölkerungsteils, allein über die Ausdehnung des Wahlrechts zu entscheiden, umstritten sein. Die korrekte Bestimmung des Demos muss daher immer schon erfolgt sein, bevor legitime Mehrheitsentscheidungen getroffen werden können. In den Worten von Frederick Whelan (1983, 15): „Determination of the criteria or bounds of the citizen body [...] is a matter that is logically prior to the operation of the majority principle and cannot be solved by it.“

Die demokratietheoretische Diskussion des Demos-Problems hat sich zunächst auf die Berechtigung zur Teilnahme an Wahlen auf der staatlichen Ebene konzentriert. Im Blickpunkt stand vor allem der Ausschluss wichtiger Bevölkerungsgruppen aus dem politischen Prozess, der die Frühphase moderner Demokratien geprägt hat (Beckman 2009, 6-10). Neben der bereits erwähnten rassistischen Diskriminierung, die Schwarze unter anderem in Südafrika und den USA erfahren haben, stechen historisch zwei weitere Formen der Exklusion hervor. Zum einen war das Stimmrecht in vielen Fällen ganz oder teilweise an die Eigentumsverhältnisse der Bürger gekoppelt. Personen ohne Grundeigentum und Vermögen, wie z. B. viele Industriearbeiter, konnten nicht oder nur in begrenztem Umfang an der Selbstgesetzgebung der demokratischen Gemeinschaft teilnehmen. ${ }^{3}$ Zum anderen war die

3 Siehe hierzu die klassischen Rechtfertigungsversuche von Immanuel Kant (1991, 150-153) und John Stuart Mill (1991, 169-195). 
Möglichkeit, politische Repräsentanten zu wählen, zunächst ausschließlich dem männlichen Bevölkerungsteil vorbehalten. Die meisten demokratischen Staaten haben erst zu Beginn des 20. Jahrhunderts - die Schweiz erst 1971 - nach langen gesellschaftlichen Kontroversen das Frauenwahlrecht eingeführt.

Der wohl einflussreichste Vorschlag zur Lösung des Demos-Problems kann als unmittelbare Reaktion auf die als illegitim empfundene Ausgrenzung der genannten Bevölkerungsgruppen verstanden werden. Schon Dahl hat dafür plädiert, sich bei der Vergabe politischer Partizipationsrechte danach zu richten, wer den gesetzlichen Maßnahmen unterliegt und insofern staatlichem Zwang ausgesetzt ist. In „Democracy and its Critics“ schreibt Dahl (1989, 127): „Every adult subject to a government and its laws must be presumed to be qualified as, and has an unqualified right to be, a member of the demos.“ Das so genannte „subject-to-coercion principle“ beruft sich auf die Notwendigkeit, die Einschränkung der individuellen Autonomie zu rechtfertigen, die mit der Androhung oder Ausübung von Zwang verbunden ist. Die spezifisch demokratische Form der Legitimation bestehe darin, die zwangsunterworfenen Personen an den politischen Entscheidungen zu beteiligen und sie so zu Mitautoren der staatlichen Gesetze zu erheben (Abizadeh 2008, 39-42). Folglich müsse die Gruppe der Personen, auf die die staatlichen Gesetze Anwendung finden, mit der Gruppe der stimmberechtigten Personen identisch sein.

Im Kontext der kommunalen Online-Partizipation, die im Fokus des vorliegenden Beitrags steht, sieht sich die Bestimmung des Demos in verschiedener Hinsicht mit Bedingungen konfrontiert, die in der bisherigen Diskussion nicht oder nicht ausreichend reflektiert wurden. Zum einen sind die Ergebnisse der Online-Verfahren, die auf städtischer Ebene durchgeführt werden, rechtlich nicht verbindlich. Dadurch entsteht die Frage, ob Verfahren, die einen rein konsultativen Charakter haben, denselben Rechtfertigungsanforderungen genügen müssen wie die eigentlichen Entscheidungsprozesse. Zum anderen bezieht sich die virtuelle Bürgerbeteiligung häufig auf städtische Maßnahmen, die nicht mit Zwangsandrohung oder -anwendung im engeren Sinne einhergehen. Bei Verfahren, wie den im vorigen Abschnitt angesprochenen Raddialogen, bietet das „subject-to-coercion principle“ daher keine Orientierungshilfe. ${ }^{4}$ Schließlich erscheint der Ein-

4 Das „subject-to-coercion principle“ begegnet bereits in der bestehenden, an Offline-Verfahren orientierten Diskussion ernsten Einwänden, die aber im 
satz virtueller Instrumente gerade deshalb attraktiv, weil er eine einfache Möglichkeit der politischen Teilhabe für eine sehr große Zahl von Menschen bietet. Die kommunale Online-Partizipation neigt daher zu einem weitgehenden Verzicht auf Zugangsbeschränkungen und wirft legitimationstheoretische Fragen auf, die sich von der klassischen Exklusionsproblematik grundlegend unterscheiden.

Der Schwerpunkt der weiteren Überlegungen liegt auf den normativen Problemen, die sich aus der Offenheit kommunaler Online-Verfahren ergeben. Im folgenden Abschnitt wird die These vertreten, dass ein vollständiger oder weitgehender Verzicht auf Zugangsbeschränkungen zu einer unplausiblen „Überdehnung“ des Demos führt. Demnach sind die meisten digitalen Beteiligungsinstrumente, die gegenwärtig auf kommunaler Ebene zum Einsatz gelangen, mit einem gravierenden Legitimationsdefizit behaftet. Perspektivisch stellt sich somit die schwierige Aufgabe, geeignete Kriterien für die Gewährung von Teilhaberechten und die Begrenzung des Demos zu entwickeln. Im vorliegenden Aufsatz soll aber zunächst nur die grundlegende Notwendigkeit, den Zugang zu kommunalen Online-Verfahren zu regeln, begründet und gegen Einwände verteidigt werden.

\section{4. Über-Inklusivität als Problem der Online-Partizipation}

Das wohl wichtigste Motiv für die Offenheit vieler kommunaler Online-Instrumente dürfte das Bestreben sein, die demokratischen Prozesse durch die Einbeziehung möglichst vieler Bürgerinnen und Bürger zu beleben. Je mehr Personen die Partizipationschancen nutzen, die das Internet bietet, desto höher - so die verbreitete Einschätzung - sei die Legitimität und Akzeptanz der getroffenen Entscheidungen. Zudem mag es bei unverbindlichen Verfahren, die die letzte Entscheidung bei den gewählten Repräsentanten belassen, so aussehen, als sei eine Beschränkung des Zugangs nicht erforderlich. Da es primär um die Information und Beratung der Abgeordneten geht, scheine es nicht ratsam zu sein, von vornherein auf den möglicherweise gewinnbringenden Input bestimmter Personen(gruppen) zu verzichten. Eine nicht zu unterschätzende Rolle dürften ferner die Datenschutzprobleme spielen, die unvermeidlich auftreten, sobald Partizipationsrechte definiert und Zugänge kontrolliert werden. Wenn im Rahmen von Online-Verfahren für die Teil-

Rahmen des vorliegenden Artikels nicht erörtert werden können. Siehe hierzu Saunders (2011a, 71-73) und Bauböck (2015, 821-824). 
nahme eine Registrierung notwendig ist, besteht die Gefahr einer Re-Identifikation von anonymisierten Diskussionsbeiträgen und Stimmverhalten. Offene Verfahren, die auf eine persönliche Anmeldung verzichten, müssen sich weitaus weniger mit Fragen des Datenschutzes und diesbezüglicher Befürchtungen in der Bevölkerung auseinandersetzen.

Wenn eine Kommune die Partizipationsrechte an den von ihr initiierten Online-Verfahren nicht einschränkt, kann die Teilnahme ganz unabhängig vom Wohnort erfolgen. So kann sich z. B. bei einem Raddialog der Stadt Flensburg auch eine Bürgerin aus Saarlouis, Rosenheim oder Görlitz zu Wort melden. Da das Internet keine nationalen Grenzen kennt, steht im Prinzip sogar Personen, die in anderen Ländern beheimatet sind, die Mitwirkung offen. Gegenüber der klassischen Diskussion, die im Fokus des vorigen Abschnitts stand, verändert sich damit die Problemlage bei der Konstitution des Demos grundlegend. Traditionelle Offline-Verfahren haben häufig auf Grund ihrer Über-Exklusivität, d.h. der Nichtberücksichtigung von Personen(gruppen), die einen plausiblen Anspruch auf politische Beteiligung geltend machen können, Kritik auf sich gezogen. Hingegen erscheint die Legitimität der neuen Online-Verfahren auf Grund ihrer Über-Inklusivität, d.h. der Einbeziehung von Personen(gruppen), die keinen unmittelbar einsichtigen Anspruch auf politische Mitwirkung haben, zweifelhaft.

Der im vorigen Abschnitt erwähnte empirische Befund einer häufig schwach ausgeprägten Bereitschaft, an kommunalen Online-Verfahren teilzunehmen, spricht nicht gegen die normative Relevanz der hier erörterten Thematik. Eine legitimationstheoretisch problematische Über-Inklusivität besteht immer dann, wenn Personen ein Partizipationsrecht erhalten, die nicht sinnvoll dem Demos zugerechnet werden können. Insofern geht es nicht um die Anzahl der Personen, die faktisch an einem Verfahren teilnehmen, sondern um die adäquate Zusammensetzung der jeweiligen Beratungsoder Abstimmungsgemeinschaft. Ein digitales Partizipationsverfahren kann auch dann über-inklusiv sein, wenn es auf geringes Interesse stößt und nur wenige Personen zur Mitwirkung motiviert. Die niedrige Teilnahmequote, die viele kommunale Online-Verfahren aufweisen, wirft eigene legitimationstheoretische Fragen auf, die sich unabhängig vom Problem der Über-Inklusivität stellen (und hier nicht erörtert werden).

Eine grundlegende Schwierigkeit für die Diskussion von Über-Inklusivität ergibt sich aus dem Dissens, der in der modernen Demokratietheorie über die Rechtfertigung demokratischer Verfahren besteht. Je nachdem, wie die Legitimation demokratischer Verfahren begründet wird, verschiebt sich 
der Blick auf die Anforderungen, die an die Komposition des Demos gestellt werden müssen. So werden z.B. Theorien, die auf die besondere Eignung demokratischer Verfahren abstellen, zu „korrekten“ Entscheidungen zu führen, Wert auf die Sachkompetenz der Teilnehmer legen. Aus ihrer Perspektive ist ein Verfahren dann über-inklusiv, wenn es uninformierte Personen einbezieht, deren Mitwirkung die Qualität der Beratung oder Beschlussfassung zu senken droht. Hingegen werden Theorien, die das Ziel demokratischer Verfahren darin sehen, allen von einer Entscheidung betroffenen Personen ein Mitspracherecht einzuräumen, vor allem fragen, wessen Interessen berührt sind. Entsprechend ist ein demokratisches Verfahren dann als über-inklusiv zu bewerten, wenn es Personen die Möglichkeit der Partizipation eröffnet, die die Entscheidung in keiner Weise betrifft. Schließlich werden Theorien, die demokratische Prozesse als Ausdruck der kollektiven Selbstbestimmung verstehen, einen starken Fokus auf die Zugehörigkeit zu der politischen Gemeinschaft legen, die über das Selbstbestimmungsrecht verfügt. Über-Inklusivität besteht aus dieser Sicht immer dann, wenn Außenstehende mit Partizipationsrechten ausgestattet werden, wodurch ein Element der Fremdbestimmung Eingang in die Verfahren findet, das dem demokratischen Ideal widerspricht. ${ }^{5}$

Die grundlegende Kontroverse zwischen den verschiedenen Legitimationstheorien kann im Rahmen des vorliegenden Aufsatzes nicht vertieft werden. Ungeachtet der unterschiedlichen Perspektiven, die die Legitimationstheorien auf das Phänomen der Über-Inklusivität haben, kann aber die oben formulierte Kritik am Verzicht auf Zugangsregelungen aufrechterhalten werden. Denn gleichgültig, welche Legitimationstheorie man vertritt, wird man immer die Über-Inklusivität der meisten digitalen Beteiligungsverfahren, die gegenwärtig auf kommunaler Ebene zum Einsatz gelangen, konstatieren müssen. Die Offenheit der Verfahren gibt Personen die Gelegenheit zur Mitwirkung, die über keine einschlägige Sachkenntnis verfügen. Zudem erlaubt sie Personen, an den demokratischen Prozessen teilzunehmen, deren Interessen durch den Gegenstand der Deliberation oder Beschlussfassung in

Auf die Richtigkeit von Entscheidungen stellen unter anderem Cohen (1986), Estlund (2008) und Peter (2009) ab; auf das Prinzip der Betroffenheit (all-affected-interest principle) berufen sich z. B. Goodin (2007), Agné (2010) und Scherz (2013); der Wert der kollektiven Selbstbestimmung wird unter anderem von Altman und Wellman (2009) sowie Stilz (2019) betont. Einen informativen Überblick über verschiedene Vorschläge zur Lösung des Demos-Problems bietet Cheneval (2015, 86-105). 
keiner Weise tangiert sind. Ferner können Personen Einfluss auf den Ausgang der Verfahren nehmen, die nicht der politischen Gemeinschaft angehören, die ihr Recht auf kollektive Selbstbestimmung ausübt. Folglich ergibt sich vom Standpunkt aller einschlägigen Legitimationstheorien die Notwendigkeit, das Problem der Über-Inklusivität ernst zu nehmen und geeignete Kriterien für die Begrenzung des Zugangs festzulegen.

Gegen die These, der Verzicht auf Zugangsregelungen führe zu einer problematischen Über-Inklusivität demokratischer Verfahren, gilt es zwei allgemeine Einwände zu prüfen, die als epistemisches Argument und als Indifferenzargument bezeichnet werden können. Darüber hinaus bedarf im folgenden Abschnitt - ein weiterer Kritikpunkt der Erörterung, der sich speziell aus dem konsultativen Charakter kommunaler Online-Instrumente ergibt.

Das epistemische Argument weist darauf hin, dass durch eine Begrenzung des Rechts, an demokratischen Online-Verfahren teilzunehmen, wichtige Gesichtspunkte verloren gehen können. Je mehr Perspektiven einbezogen werden, desto größer - so die leitende Überlegung - ist die Wahrscheinlichkeit, zu einer sachgerechten Entscheidung zu kommen. Demnach ist es verfehlt, die digitalen Beteiligungsinstrumente, die auf kommunaler Ebene praktiziert werden, als über-inklusiv zu bezeichnen, weil epistemische Gründe für die Konstitution eines globalen Demos sprechen. Als klassischer Bezugspunkt dienen die Erkenntnisse von Marquis de Condorcet (1976), der sich schon im 18. Jahrhundert mit der epistemischen Qualität von Entscheidungsprozessen befasst hat. In seinem berühmten Jury-Theorem hat er nachgewiesen, dass sich (unter bestimmten Bedingungen) die Wahrscheinlichkeit, in einem Mehrheitsverfahren einen korrekten Beschluss zu fassen, mit der Anzahl der beteiligten Personen erhöht. Insofern die Inklusivität demokratischer Verfahren maßgeblich dazu beitragen kann, bessere Ergebnisse zu erzielen, erscheint ein möglichst weitgehender Verzicht auf Zugangsbeschränkungen geboten.

Das epistemische Argument gegen die hier vertretene These der Über-Inklusivität vermag nicht zu überzeugen, weil es auf einer unplausiblen Kompetenzzuschreibung beruht. Bereits das Jury-Theorem von Condorcet setzt voraus, dass sich die Teilnehmer an einem Mehrheitsverfahren mit einer Wahrscheinlichkeit von mehr als 50 Prozent für die richtige Option entscheiden. Die Mitwirkung von Personen, deren Kompetenz unterhalb der genannten Schwelle liegt, verschlechtert die Aussicht, gute Ergebnisse zu erzielen. Je weiter nun eine Person von einem Gegenstand entfernt und je 
geringer ihr Wissensstand ist, desto weniger ist ihr eine sachgerechte Entscheidung zuzutrauen (Estlund 2008, 15-16). So wird z. B. eine Bürgerin aus Saarlouis mangels Ortskenntnis in aller Regel nicht in der Lage sein, einen Beitrag zur Verbesserung des Radwegenetzes in Flensburg zu leisten. ${ }^{6}$ Auch in rein deliberativen Verfahren, die sich auf einen Meinungsaustausch beschränken, steht keine Qualitätsverbesserung durch eine möglichst weitgehende Öffnung und einen möglichst inklusiven Diskurs zu erwarten. Wenn sachfremde und uninformierte Beiträge einen breiten Raum einnehmen, haben es rationale Argumente schwer, wahrgenommen zu werden und den Gang der Diskussion zu bestimmen.

Im Unterschied zum epistemischen Argument erwartet das Indifferenzargument, das auf Robert E. Goodin zurückgeht, keine besseren Entscheidungen von Verfahren mit hoher Inklusivität. Es basiert auf der weniger anspruchsvollen These, dass der Verzicht auf Zugangsbeschränkungen das Ergebnis einer Deliberation oder Beschlussfassung nicht verändert. Die Entstehung von Über-Inklusivität wird zwar nicht bestritten, aber als unbedenklich erachtet, weil sie letztlich folgenlos bleibt. Nach Goodins Überzeugung sind die Teilnehmer an demokratischen Prozessen typischerweise bemüht, ihre individuellen Interessen zur Geltung zu bringen. Personen, für die der zu beratende oder beschließende Gegenstand irrelevant ist, werden sich seiner Einschätzung nach willkürlich positionieren. Da bei einer zufälligen Entscheidung die Wahl aller verfügbaren Optionen gleich wahrscheinlich sei, könne man von einer statistischen Gleichverteilung aller indifferenten Personen ausgehen, die das Ergebnis nicht beeinflusse. Goodin (2007, 58-59) schreibt:

Suppose that people are required to vote on some issue that does not affect their interest. How will they vote? Randomly [...]. It makes no difference to the political outcome, therefore, if we enfranchise people whose interests are not affected. All that we have to worry about is ensuring the inclusion of everyone whose interests are affected.

Goodins Indifferenzargument kann aus zwei Gründen die eingangs skizzierten Bedenken gegen die Über-Inklusivität partizipativer Online-Verfahren nicht entkräften (Saunders 2011b, 284-286). Zum einen ist die Vergabe von

6 Die hier dargelegte Argumentation wendet sich nur gegen unbegrenzte Teilnahmemöglichkeiten an demokratischen Verfahren und schließt somit die Einbeziehung externer Experten nicht grundsätzlich aus. 
Teilhaberechten an Personen, die klarerweise nicht zur politischen Gemeinschaft gehören, zumindest aus Sicht einiger Legitimationstheorien auch dann problematisch, wenn sich ihre Voten gegenseitig aufheben. Die Partizipation von unbeteiligten Dritten ist - ganz unabhängig von den Folgen - mit dem Grundgedanken der individuellen wie auch der kollektiven Autonomie unvereinbar. So würde es z. B. der individuellen Autonomie von Person A widersprechen, wenn sie in allen wichtigen Entscheidungen die Meinung von Person B und Person C einbeziehen und einen Mehrheitsbeschluss herbeiführen müsste. Das gilt auch dann, wenn die Personen B und C hoffnungslos zerstritten sind und mit großer Zuverlässigkeit immer genau entgegengesetzte Positionen beziehen. Obwohl Person A - sofern nur zwei Optionen vorliegen - stets den Ausgang bestimmen könnte, wäre ihre Autonomie bereits durch die Notwendigkeit beeinträchtigt, das Votum der Personen B und $\mathrm{C}$ einholen oder gar akzeptieren zu müssen. Ähnlich verhält es sich mit der kollektiven Autonomie, wenn das Stimmrecht nicht auf die Angehörigen der politischen Gemeinschaft beschränkt bleibt.

Zum anderen ist Goodins Annahme einer Gleichverteilung der Positionen, die indifferente Personen zum Gegenstand einer Beratung oder Entscheidung einnehmen, unplausibel. Selbst wenn keine eigenen Interessen auf dem Spiel stehen, können irrelevante Aspekte, wie die Eingängigkeit eines Slogans oder die physische Attraktivität eines politischen Akteurs, in den Vordergrund treten und zu systematischen Verzerrungen führen. Ferner neigen Personen, die von den Folgen einer Entscheidung nicht betroffen sind, unter Umständen dazu, ihre Unterstützung für abstrakte Werte wie z.B. Umwelt- oder Denkmalschutz zum Ausdruck zu bringen. Sie werden möglicherweise sehr leichtfertig der lokalen Bevölkerung Lasten aufbürden, die sie in vergleichbaren Fällen nicht bereit wären, selbst zu tragen. Insofern erscheint es höchst ungewiss, ob die Erwartung sich gegenseitig aufhebender Voten, auf die Goodin seine Argumentation stützt, der Realität standhält.

\section{Konsultative Verfahren}

Wie bereits im zweiten Abschnitt dargelegt, sind die Resultate der Online-Partizipation rechtlich unverbindlich, d.h., die formale Entscheidungskompetenz verbleibt beim Stadtrat. Oftmals geäußerte Zusicherungen einer besonderen Berücksichtigung oder gar Umsetzung der Ergebnisse stellen bloß Selbstverpflichtungen dar, die keinen Rechtsanspruch erzeugen. Deshalb liegt die Frage nahe, warum der Zusammensetzung des Demos über- 
haupt Bedeutung zukommen soll. Schließlich wird die jeweilige Entscheidung letztlich vom Stadtrat und somit von einer Institution gefällt, die über demokratische Wahlen abschließend legitimiert und autorisiert ist. Diesem Einwand folgend wäre der Verzicht auf Zugangsbeschränkungen legitimationstheoretisch nicht weiter relevant und der Befund der Über-Inklusivität unproblematisch.

Tatsächlich beschäftigt sich die wissenschaftliche Literatur zum Demos-Problem fast ausschließlich mit Partizipationsrechten im Kontext verbindlicher Verfahren. Sie geht dabei von der Überlegung aus, dass die Zusammensetzung des Demos normativ relevant ist, weil sie sich auf das Ergebnis der Entscheidungen auswirken kann. Je nachdem welche Personen bzw. Personengruppen zur Teilnahme berechtigt sind, kann sich das Resultat der Beschlussfassung verändern. Die Entgegnung auf den oben skizzierten Einwand stützt sich auf die Annahme, dass sich verbindliche und unverbindliche Verfahren in dieser Hinsicht nicht grundlegend unterscheiden. Auch unverbindliche Beteiligungsverfahren lassen einen - mehr oder minder starken - Einfluss auf die Entscheidungen erwarten, die von den politischen Repräsentanten getroffen werden. Insofern hat die Zusammensetzung des Demos und speziell das Problem der Über-Inklusivität für die konsultativen Beteiligungsinstrumente, die auf kommunaler Ebene praktiziert werden, sehr wohl legitimationstheoretische Bedeutung.

Die Vermutung, dass konsultative Verfahren nicht folgenlos bleiben, ist schon auf Grund ihrer Zielsetzung naheliegend. Denn die Motivation, den Bürgerinnen und Bürgern die Möglichkeit der Beteiligung zu eröffnen, besteht ja gerade darin, zu besseren Entscheidungen zu gelangen, die mit einer höheren Akzeptanz rechnen können. Entsprechend heben viele kommunale Leitlinien ihre Absicht hervor, die Ergebnisse der konsultativen Verfahren angemessen zu berücksichtigen. Zwar äußern sich die Kommunen unterschiedlich zu der Frage, für wie verbindlich sie den Ausgang von Beteiligungsverfahren erachten, und sehen häufig von expliziten Versprechungen ab. Während z.B. die Stadt Wolfsburg nur zusichert, „die Ergebnisse des Bürgermitwirkungsverfahrens in öffentlichen Sitzungen zu diskutieren“ (Stadt Wolfsburg 2015, 17), ist „[g]ute Bürgerbeteiligung in Wuppertal [...] verbindlich und verlässlich im Anliegen, im Verlauf und in den Ergebnissen“ (Stadt Wuppertal 2017, 8). Doch selbst in Wolfsburg ist von der „Mitwirkung von Bürgerinnen und Bürgern in politischen Entscheidungsprozessen“ (Stadt Wolfsburg 2015, 19) die Rede, was nur dann Sinn ergibt, wenn ein Einfluss nicht prinzipiell ausgeschlossen wird. Eine typische Stellungnah- 
me findet sich in den Leitlinien von Berlin-Mitte: „Gute Bürgerbeteiligung setzt auf Verbindlichkeit. Die erarbeiteten Ergebnisse werden verbindlich und verlässlich umgesetzt, bzw. erläutert, welche Gründe dem entgegenstehen“ (Bezirksamt Mitte von Berlin 2017, 4). Demnach ist die Nichtbeachtung konsultativer Verfahren nur dann vorgesehen, wenn gute Gründe, wie etwa mangelnde Qualität oder Aussagekraft, gegen die Umsetzung ihrer Ergebnisse sprechen.

Insofern letztlich die Gemeinderäte als gewählte Repräsentativorgane darüber entscheiden, ob und inwieweit die Ergebnisse partizipativer Verfahren in staatliche Entscheidungen eingehen, rückt deren Verhältnis zu den Repräsentierten ins Zentrum der Betrachtung. Dabei sagen das freie Mandat und die formale Unverbindlichkeit konsultativer Verfahren noch nicht allzu viel über das tatsächliche Handeln der Abgeordneten aus. Erstens lässt die formale Entscheidungsautonomie der Abgeordneten Raum für nahezu jeden möglichen Umgang mit den Ergebnissen, von der gänzlichen Missachtung bis hin zur vollständigen Umsetzung (Rehfeld 2009). Zweitens steht der Umgang repräsentativer Entscheidungsorgane mit den Ergebnissen der Bürgerbeteiligung trotz der ihnen formal zugesicherten Unabhängigkeit im Fokus öffentlicher Bewertung und Kritik (Urbinati 2006). Somit besteht die eigentliche Frage darin, ob und inwieweit sich Repräsentantinnen und Repräsentanten de facto in ihrer Entscheidung bezüglich des Verfahrensgegenstandes an den Ergebnissen der Bürgerbeteiligung orientieren (Rehfeld 2009).

Innerhalb der Ideengeschichte findet sich eine lange Diskussion, die sich der Rolle der Repräsentantinnen und Repräsentanten und den Normen, die deren Handeln anleiten sollen, widmet (Pitkin 1967; Manin 1997; Mansbridge 2003; Urbinati 2006). Besonders prominent wird innerhalb dieser Diskussion die sogenannte Trustee-Delegate-Kontroverse verhandelt, die für die hier zu erörternde Thematik unmittelbar einschlägig ist. Während das Rollenbild des Repräsentanten als Treuhänder (Trustee) dessen Aufgabe darin sieht, nach eigenem Wissen und Gewissen zu entscheiden, verpflichtet das Rollenbild des Repräsentanten als Delegierten (Delegate) diesen darauf, in seinen Entscheidungen den Willen seiner Wähler umzusetzen.7 In den Po-

$7 \quad$ Bereits Pitkin (1967) hat darauf hingewiesen, dass dieser strikte Dualismus schon auf theoretischer Ebene nicht zu halten sei, da das Konzept politischer Repräsentation weder ohne eine gewisse Abhängigkeit noch ohne eine gewisse Unabhängigkeit der Repräsentanten von den Präferenzen der Bürger zu 
litikwissenschaften wird eine solche Orientierung an dem Willen der Bürger als politische Responsivität bezeichnet (Powell 2004). Staatliches Handeln ist umso responsiver, je mehr es dem Willen entspricht, den die Bürgerinnen und Bürger im Rahmen der Online-Partizipation oder über andere Kanäle artikulieren.

Auch wenn der Wert politischer Responsivität innerhalb der politischen Philosophie durchaus umstritten ist, ist er im populären Verständnis der Demokratie als der Herrschaft des Volkes fest verankert. Er begründet die weitverbreitete Forderung, dass sich gewählte Mandatsträgerinnen und Mandatsträger in ihren Entscheidungen maßgeblich am Bürgerwillen und somit an der Rollenkonzeption des Delegierten orientieren sollten: „The mandate [bzw. delegate] conception of representation is widespread: scholars, journalists and ordinary citizens rely on it as if it were axiomatic.“ (Manin et al. 1999, 30). Responsivitätserwartungen können sowohl Teil des eigenen Rollenverständnisses der Abgeordneten sein als auch von den Wählern an diese herangetragen werden. Zum einen können die Abgeordneten intrinsisch motiviert sein, die Ergebnisse konsultativer Verfahren weitgehend umzusetzen, weil sie sich zur Beachtung des Bürgerwillens verpflichtet fühlen. Schließlich haben sie selbst die Bürgerbeteiligung veranlasst, weil sie der im repräsentativen System bestehenden Schwierigkeit, die Sichtweise der Bürgerinnen und Bürger angemessen zu kennen und zu berücksichtigen, begegnen wollten. So lassen sich aus der Stimmabgabe für Parteien mit umfassenden Wahlprogrammen nur selten akkurate Rückschlüsse auf die Präferenzen der Wählerschaft im Hinblick auf spezifische Fragen ziehen. Insofern kann die kommunale Online-Partizipation repräsentative Wahlen sinnvoll ergänzen, indem sie Bürgerinnen und Bürgern die Möglichkeit eröffnet, ihre Wünsche konkreter zu artikulieren.

Zum anderen machen die mediale Öffentlichkeit ihre Kritik und vor allem Bürger ihre Wahlentscheidungen auch vom responsiven Verhalten der Abgeordneten abhängig. Wenn zunächst partizipative Verfahren initiiert, dann aber ihre Ergebnisse ignoriert werden, entsteht leicht der Eindruck der „Bürgertäuschung“. Es sieht dann so aus, als hätte man die Bürgerinnen und Bürger nur befragt, um höhere Akzeptanz für eine Entscheidung zu erzielen,

denken sei. Zudem haben empirische Studien von Eulau und Kollegen (1959) gezeigt, dass die Kontrastierung von Treuhänder und Delegierten auch die politische Praxis nicht akkurat beschreiben kann, da die Repräsentanten je nach Kontext bemüht sind, mal als Delegierte und mal als Treuhänder zu agieren. 
die eigentlich schon feststand. Sobald die Bürgerschaft aber Präferenzen artikuliert, die den Vorstellungen der Repräsentanten entgegenstehen, ist man nicht bereit, das politische Handeln an ihnen auszurichten. Dadurch steigt der politische Druck, den Ergebnissen der kommunalen Online-Partizipation zu entsprechen und allenfalls dann abweichende Entscheidungen zu treffen, wenn sich sehr starke Rechtfertigungsgründe anführen lassen. Somit spricht also viel dafür, dass auch formal unverbindliche Beteiligungsverfahren oftmals einen Einfluss auf legitimierungsbedürftige staatliche Entscheidungen ausüben. Folglich ist die Zusammensetzung des Demos auch im Kontext der kommunalen Online-Partizipation legitimationstheoretisch relevant und der weitgehende Verzicht auf Zugangsbeschränkungen als problematisch einzustufen.

Leider liegen keine empirischen Studien vor, die sich über Einzelfälle hinaus mit dem Einfluss von Bürgerbeteiligung auf staatliche Entscheidungen beschäftigen. Die Untersuchung von Einzelfällen in der wissenschaftlichen Literatur zeigt eine unterschiedlich weitgehende Umsetzung der Ergebnisse konsultativer Verfahren (Neunecker 2016, 57). Dennoch gibt es einige Untersuchungen, die die vorstehend angestellten Überlegungen zur Responsivität von Repräsentanten unterstützen. So hat z. B. in einer repräsentativen Umfrage aus dem Jahre 2010 über die Hälfte der Befragten in Deutschland die Auffassung vertreten, dass Bundestagsabgeordnete bei Entscheidungen (meistens) der Meinung der Wähler folgen sollten. Nur zehn Prozent der Befragten gaben an, dass die Abgeordneten (meistens) gemäß der eigenen Meinung handeln sollten (Dageförde 2013). Im kommunalen Kontext zeigt eine Studie von Wilker (2019, 242), dass für Ratsmitglieder bei strittigen Entscheidungen die eigene Position, die Position der direkt betroffenen Bürger und die Position der Wähler nahezu gleichermaßen wichtige Bezugspunkte darstellen. Auch wenn die Datenlage unvollständig (und mitunter widersprüchlich) ist, gibt es letztlich doch starke Indizien, dass Responsivitätserwartungen im Kontext kommunaler Bürgerbeteiligung eine große Rolle spielen und die Ergebnisse konsultativer Verfahren von hoher praktischer Relevanz für die Entscheidungsfindung des Stadtrats sind.

Abschließend gilt es noch zwei möglichen Missverständnissen vorzubeugen. Erstens treffen die vorstehenden Überlegungen keine Aussage darüber, ob (und ggf. unter welchen Umständen) es wünschenswert ist, digitale Beteiligungsverfahren durchzuführen. Ziel des Aufsatzes ist es lediglich, auf ein Legitimationsproblem aufmerksam zu machen, das durch die offene Form entsteht, in der die kommunale Online-Partizipation gegenwär- 
tig praktiziert wird. Die Frage, inwieweit das Instrumentarium der digitalen Bürgerbeteiligung auf städtischer Ebene genutzt werden soll, muss hier nicht beantwortet werden. Es geht nur darum zu zeigen, dass eine Auseinandersetzung mit der Komposition des Demos erforderlich wird, sobald man sich für die Initiierung digitaler Beteiligungsverfahren entscheidet. Zweitens wird keine Aussage darüber getroffen, ob (und ggf. in welchem Umfang) ein responsives Verhalten der politischen Repräsentanten normativ geboten ist. Das Problem der Über-Inklusivität, das hier im Fokus steht, ergibt sich schon aus der Annahme, dass unverbindliche Beteiligungsverfahren das Verhalten politischer Entscheidungsträgerinnen und -träger faktisch beeinflussen. Die Frage, ob ein solcher Einfluss wünschenswert ist und Abgeordnete als Delegierte agieren sollten, ist davon unabhängig und nicht Gegenstand der vorliegenden Untersuchung.

\section{Resümee}

Auch im modernen Nationalstaat und unter den Bedingungen globaler Vernetzungsmöglichkeiten durch das Internet haben die Städte ihre politische Bedeutung nicht eingebüßt. Ganz im Gegenteil haben sie sich in jüngster Zeit zu einem Experimentierfeld für innovative Konzepte der Bürgerbeteiligung entwickelt, die einen wichtigen Beitrag zur Stärkung der Demokratie leisten können. Die neu eingerichteten Verfahren kommunaler Online-Partizipation müssen allerdings, um soziale Akzeptanz erzielen zu können, basalen Kriterien der Legitimität genügen. Ein wichtiger und noch wenig erörterter Aspekt stellt dabei die Vergabe von Teilhaberechten dar, die im Fokus der vorliegenden Untersuchung stand. Wie die obigen Überlegungen gezeigt haben, führt der weitgehende Verzicht auf Zugangsbeschränkungen, der die gegenwärtig praktizierten Teilhabeinstrumente kennzeichnet, zu einer legitimationstheoretisch problematischen Über-Inklusivität.

Die Einwände, die gegen die These der Über-Inklusivität angeführt werden können, haben sich als nicht stichhaltig erwiesen. Erstens spricht das Ziel, die Qualität der Entscheidungen zu verbessern, die auf kommunaler Ebene getroffen werden, nicht für eine „Entgrenzung“ des Demos. Im Gegenteil sind sachgerechtere Entscheidungen zu erwarten, wenn Personen, die keinen Bezug zu den betreffenden Angelegenheiten haben, die Teilnahme verwehrt bleibt. Zweitens gibt es gute Gründe für die Annahme, dass der weitgehende Verzicht auf Zugangsbeschränkungen das Ergebnis kommunaler Online-Verfahren beeinflussen kann. Insofern vermag das von Ro- 
bert E. Goodin formulierte Indifferenzargument, das die Beteiligung nicht betroffener Personen für folgenlos erachtet, nicht zu überzeugen. Drittens ist der Verweis auf den unverbindlichen Charakter der digitalen Teilhabeinstrumente, die auf kommunaler Ebene praktiziert werden, nicht einschlägig. Da die Ergebnisse der Partizipationsverfahren typischerweise einen starken Einfluss auf die politischen Entscheidungsträger entfalten, bedarf die $\mathrm{Zu}-$ sammensetzung des Demos einer überzeugenden Rechtfertigung.

Im Ergebnis ist die Mehrzahl der digitalen Teilhabeverfahren, die gegenwärtig im städtischen Raum durchgeführt werden, mit einem gravierenden Legitimationsdefizit behaftet. Die Über-Inklusivität, die der weitgehende Verzicht auf Zugangsbeschränkungen hervorruft, droht das demokratische Potenzial der kommunalen Online-Partizipation zu unterminieren. Insofern stellt sich für zukünftige Forschung die Aufgabe, normative Kriterien zu begründen, an denen sich die Vergabe von Teilhaberechten orientieren soll. Das Problem der Über-Inklusivität, das vorstehend analysiert wurde, weist auf die Notwendigkeit hin, den Demos kommunaler Beteiligungsverfahren einzugrenzen. Daraus kann man aber nicht ohne Weiteres schließen, dass ausschließlich Personen über Partizipationsrechte verfügen sollten, die ihren Wohnsitz in der betreffenden Kommune haben. Ernsthaft zu prüfen sind Kriterien, die eine bedingte - auf Sacherwägungen gestützte - Öffnung kommunaler Online-Instrumente empfehlen. So erscheint es etwa bedenkenswert, Personen, die außerhalb der Kommune leben, aber von einem Verfahrensgegenstand in relevanter Weise betroffen sind, Partizipationsrechte zu gewähren. Beispielsweise mag ein Raddialog zu ausgewogeneren Ergebnissen führen, wenn auch Pendler, die die städtischen Verkehrswege mitbenutzen, ihre Interessen artikulieren können. Ob und inwieweit eine sachbezogene Erweiterung des Demos über die städtische Bevölkerung hinaus gerechtfertigt werden kann, bedarf aber einer eingehenden Diskussion, die hier nicht geleistet werden kann.

\section{Literatur}

Abizadeh, Arash. 2008. „Democratic Theory and Border Coercion: No Right to Unilaterally Control Your Own Border“. Political Theory 36: 37-65. https://doi. org/10.1177/0090591707310090.

Agné, Hans. 2010. „Why Democracy Must Be Global: Self-Founding and Democratic Intervention“. International Theory 2: 381-409. https://doi.org/10.1017/S 1752971910000254 . 
Altman, Andrew, und Christopher Heath Wellman. 2009. A Liberal Theory of International Justice. Oxford: Oxford University Press.

Aristoteles. 2012. Politik, herausgegeben von Eckart Schütrumpf. Hamburg: Meiner.

Arnstein, Sherry R. 1969. „A Ladder of Citizen Participation“.Journal of the American Institute of Planners 35(4): 216-224. https://doi.org/10.1080/01944366908977225.

Barber, Benjamin R. 2009 [1984]. Strong Democracy: Participatory Politics for a New Age. Berkeley: University of California Press.

Bauböck, Rainer. 2015. „Morphing the Demos into the Right Shape. Normative Principles for Enfranchising Resident Aliens and Expatriate Citizen“. Democratization 22: 820-839. https://doi.org/10.1080/13510347.2014.988146.

Beckman, Ludvig. 2009. The Frontiers of Democracy. The Right to Vote and its Limits. Basingstoke, New York: Palgrave Macmillan.

Bezirksamt Mitte von Berlin. 2017. Leitlinien für Bürgerbeteiligung im Bezirk Mitte von Berlin. https://www.berlin.de/ba-mitte/aktuelles/buergerbeteiligung/leitlinien_fuer_buergerbeteiligung.pdf (zuletzt abgerufen am 5·7.2020).

Brady, Henry E., Sidney Verba und Kay L. Schlozman. 1995. „Beyond SES: A Resource Model of Political Participation“. American Political Science Review 89: 271-294. https://doi.org/10.2307/2082425.

Cheneval, Francis. 2015. Demokratietheorien zur Einführung. Hamburg: Junius.

Crouch, Colin. 2004. Post-democracy. Cambridge: Polity.

Cohen, Joshua. 1986. „An Epistemic Conception of Democracy“. Ethics 97: 26-38. https://doi.org/10.1086/292815.

Cooke, Maeve. 2000. „Five Arguments for Deliberative Democracy“. Political Studies 48(5): 947-969.

Condorcet, Marquis de. 1976. Selected Writings, herausgegeben von K.M. Baker. Indianapolis: Bobbs-Merrill Company.

Dageförde, Mirjam. 2013. „Weit entfernt vom ,idealen Abgeordneten“? Zu Normen und Praxis parlamentarischer Repräsentation aus Sicht der Bürger". Zeitschrift für Parlamentsfragen 44(3): 580-592.

Dahl, Robert A. 1989. Democracy and Its Critics. New Haven, London: Yale University Press.

Dahl, Robert A. 1990 [1970]. After the Revolution? Authority in a Good Society. 2., überarb. Aufl., New Haven, London: Yale University Press.

Deutscher Städtetag. 2013a. Beteiligungskultur in der integrierten Stadtentwicklung: Arbeitspapier der Arbeitsgruppe Bürgerbeteiligung des Deutschen Städtetages. https://www.netzwerk-buergerbeteiligung.de/fileadmin/Inhalte/PDF-Dokumente/Meldungen/dst_beteiligungskultur_2013.pdf (zuletzt abgerufen am 27.1.2021). 
Deutscher Städtetag. 2013b. Thesen zur Weiterentwicklung lokaler Demokratie. https://www.netzwerk-buergerbeteiligung.de/fileadmin/Inhalte/PDF-Dokumente/ thesenpapier_lokale_demokratie_endfassung_ha_07_11_2013.pdf (zuletzt abgerufen am 27.1.2021).

Escher, Tobias. 2018. Bonner Rad-Dialog: Abschlussbericht zur aktiven Beteiligungsphase vom 13. September bis 18. Oktober 2017. https://www.bonn-machtmit.de/sites/default/files/unit/files/Bonner_Rad-Dialog_Abschlussbericht.pdf (zuletzt abgerufen am 5·7.2020).

Estlund, David. 2008. Democratic Authority. A Philosophical Framework. Princeton: Princeton University Press.

Eulau, Heinz et al. 1959. „The Role of the Representative: Some Empirical Observations on the Theory of Edmund Burke“. American Political Science Review 53(3): 742-756. https://doi.org/10.2307/1951941.

Goodin, Robert E. 2007. „Enfranchising All Affected Interests, and Its Alternative“. Philosophy \& Public Affairs 35: 40-68. https://doi.org/10.1111/j.1088-4963.2007.00098.x.

Habermas, Jürgen. 1992. Faktizität und Geltung: Beiträge zur Diskurstheorie des Rechts und des demokratischen Rechtsstaats. Frankfurt am Main: Suhrkamp.

Hamilton, Alexander, James Madison und John Jay. 2007 [1787/88]. Die Federalist Papers. München: Beck.

IAP2 International Association for Public Participation. 2018. IAP2 Spectrum of Public Participation. https://iap2.org.au/wp-content/uploads/2020/01/2018_ IAP2_Spectrum.pdf (zuletzt abgerufen am 23.12.2020).

Kant, Immanuel. 1991 [1793]. Über den Gemeinspruch: Das mag in der Theorie richtig sein, taugt aber nicht für die Praxis. In Immanuel Kant, Schriften zur Anthropologie, Geschichtsphilosophie, Politik und Pädagogik 1, Werkausgabe Band XI, herausgegeben von Wilhelm Weischedel, 127-172. Frankfurt am Main: Suhrkamp.

Kreager, Philip. 2008. „Aristotle and Open Population Thinking“. Population and Development Review 34: 599-629. https://doi.org/10.1111/j.1728-4457.2008.00243.x.

Manin, Bernard, Adam Przeworski und Susan Carol Stokes. 1999. Elections and Representation. In Democracy, accountability, and representation, herausgegeben von Adam Przeworski, Susan Carol Stokes und Bernard Manin, 29-54. Cambridge: Cambridge University.

Manin, Bernard. 1997. The Principles of Representative Government. Cambridge: Cambridge University Press.

Mansbridge, Jane. 2003. Rethinking Representation. The American Political Science Review 97(4): 515-528.

Masser, Kai, Adriano Pistoia und Philipp Nitzsche. 2013. Bürgerbeteiligung und Web 2.o: Potentiale und Risiken webgestützter Bürgerhaushalte. Wiesbaden: Springer V. 
McLuhan, Marshall, und Bruce R. Powers. 1992. The Global Village: Transformations in World Life and Media in the 21th Century. New York: Oxford University Press.

Mill, John Stuart. 1991 [1861]. Considerations on Representative Government. New York: Prometheus.

Neunecker, Martina. 2016. Partizipation trifft Repräsentation: die Wirkungen konsultativer Bürgerbeteiligung auf politische Entscheidungen. Wiesbaden: Springer V.

Pitkin, Hanna F. 1967. The Concept of Representation. Berkeley, Los Angeles: University of California Press.

Pateman, Carole. 1970. Participation and democratic theory. Cambridge: Cambridge University Press.

Peter, Fabienne. 2009. Democratic Legitimacy. Abingdon, New York: Routledge.

Powell, G. Bingham. 2004. „The Chain of Responsiveness“. Journal of Democracy 15(4): 91-105. https://doi.org/10.1353/jod.2004.0070.

Rehfeld, Andrew. 2009. „Representation Rethought: On Trustees, Delegates, and Gyroscopes in the Study of Political Representation and Democracy“. American Political Science Review 103(2): 214-230. https://doi.org/10.1017/ Soo03055409090261.

Rousseau, Jean-Jacque. 2011 [1762]. Vom Gesellschaftsvertrag: oder Prinzipien des Staatsrechts. Stuttgart: Reclam.

Ruesch, Michelle. 2018. empatia: D3.2 Pilots implementation - final. https:// www.wuppertal.de/microsite/buergerbeteiligung/verfahren/content/Buergerhaushalt.php.media/273094/EMPATIA_Evaluation.pdf (zuletzt abgerufen am 26.5.2020).

Saunders, Ben. 2011a. „Immigration, Rights and Democracy“. Theoria. A Journal of Social and Political Theory 58: 58-77. https://doi.org/10.3167/th.2011.5812905.

Saunders, Ben. 2011b. „Defining the Demos“. Politics, Philosophy \& Economics 11: 280-301. https://doi.org/10.1177/1470594X11416782.

Scherz, Antoinette. 2013. „The Legitimacy of the Demos: Who Should be Included in the Demos and on What Grounds?" Living Reviews in Democracy 4: 1-14. https://doi.org/10.5167/uzh-91127.

Senatsverwaltung für Stadtentwicklung und Wohnen. 2019. Leitlinien für Beteiligung von Bürgerinnen und Bürgern an Projekten und Prozessen der räumlichen Stadtentwicklung. https://leitlinien-beteiligung.berlin.de/documents/332/2019 Broschure_Leitlinien_fur_Burgerbeteiligung_an_der_Stadtentwicklung.pdf (zuletzt abgerufen am 11.7.2020).

Stadt Jena. 2016. Leitlinien für Bürgerbeteiligung in Jena. https://beteiligung.jena. de/sites/default/files/2019-03/Leitlinien_01082017_geschuetzt.pdf (zuletzt abgerufen am 15.1.2021). 
Stadt Wolfsburg. 2015. Konzept: BÜRGERmitWIRKUNG WOLFSBURG. https:// www.wolfsburg.de/ /media/wolfsburg/statistik_daten_fakten/buergerbeteiligung/mitwirkung/konzept-mitwirkung.pdf (zuletzt abgerufen am 21.1.2020).

Stadt Wuppertal. 2017. Leitlinien Bürgerbeteiligung Wuppertal. https://www.wuppertal.de/microsite/buergerbeteiligung/medien/bindata/2018_leitlinien_A5_ web.pdf (zuletzt abgerufen am 11.7.2020).

Stilz, Anna. 2019. Territorial Sovereignty. A Philosophical Exploration. Oxford: Oxford University Press.

Tocqueville, Alexis de. 2014 [1835/40]. Über die Demokratie in Amerika. Stuttgart: Reclam.

Urbinati, Nadia. 2006. Representative democracy: principles and genealogy. Chicago: University of Chicago Press.

Whelan, Frederick G. 1983. „Prologue: Democratic Theory and the Boundary Problem“. Nomos 25: 13-47.

Wilker, Nadja. 2019. Online-Bürgerbeteiligung und politische Repräsentation. Wiesbaden: Springer Fachmedien Wiesbaden.

Wilker, Nadja, Sabrina Schöttle, Malte Steinbach, Theresa Witt und Peter Gladitz. 2016. „Monitor Online-Partizipation: Ein Drittel aller Städte und Gemeinden in NRW setzt E-Partizipation ein“. eNewsletter Wegweiser Bürgergesellschaft (11): 1-5.

Zepic, Robert, Marcus Dapp und Helmut Krcmar. 2017. „E-Partizipation und keiner macht mit“. HMD Praxis für Wirtschaftsinformatik 54: 488-501. https://doi. org/10.1365/s40702-017-0328-z. 
